\title{
Luteolin potentiates the effects of aminoglycoside and $\beta$-lactam antibiotics against methicillin-resistant Staphylococcus aureus in vitro
}

\author{
DAE-KI JOUNG $^{1 *}$, OK-HWA KANG ${ }^{1 *}$, YUN-SOO SEO ${ }^{1}$, TIAN ZHOU ${ }^{1}$, YOUNG-SEOB LEE ${ }^{2}$, SIN-HEE HAN $^{2}$, \\ SU-HYUN MUN ${ }^{3}$, RYONG KONG ${ }^{3}$, HO-JUN SONG ${ }^{3}$, DONG-WON SHIN ${ }^{4}$ and DONG-YEUL KWON ${ }^{1}$ \\ ${ }^{1}$ Department of Oriental Pharmacy, College of Pharmacy and Wonkwang-Oriental Medicines Research Institute, \\ Wonkwang University, Iksan, Jeonbuk 570-749; ${ }^{2}$ Department of Herbal Crop Research, \\ National Institute of Horticultural \& Herbal Science, Rural Development Administration, Eumsung, Chungbuk 369-873; \\ ${ }^{3}$ BK21 Plus Team, Professional Graduate School of Oriental Medicine, Wonkwang University, Iksan, Jeonbuk 570-749; \\ ${ }^{4}$ Department of Oriental Medicine Resources, Sunchon National University, Suncheon,
} Jeonnam 540-742, Republic of Korea

Received March 17, 2015; Accepted January 15, 2016

DOI: $10.3892 /$ etm.2016.3212

\begin{abstract}
Methicillin-resistant Staphylococcus aureus (MRSA) infection has become a serious clinical problem worldwide, and alternative natural or combination drug therapies are required for its treatment. The aim of the present study was to examined the antimicrobial activity of luteolin (LUT) against MRSA. Luteolin is a polyphenolic flavonoid compound with a wide spectrum of biological activities. The antimicrobial activities of LUT and the antibiotics ampicillin (AM), oxacillin (OX) and gentamicin (GT), used alone or in combination, were evaluated against five clinical MRSA isolates and two reference strains using a minimum inhibitory concentration (MIC) assay, MTT colorimetric assay, checkerboard dilution test and time-kill assay. The MIC of LUT against all strains was found to be $62.5 \mu \mathrm{g} / \mathrm{ml}$. The combinations of LUT and antibiotics exhibited a synergistic effect against MRSA in the majority of cases, as determined by the checkerboard method. Time-kill curves revealed that a combination of LUT with AM, OX or GT significantly reduced bacterial counts, which dropped below the lowest detectable limit after $24 \mathrm{~h}$. These results indicate that LUT potentiates the effects of $\beta$-lactam and aminoglycoside antibiotics against MRSA.
\end{abstract}

Correspondence to: Professor Dong-Yeul Kwon, Department of Oriental Pharmacy, College of Pharmacy and Wonkwang-Oriental Medicines Research Institute, Wonkwang University, Shinyong-Dong 344-2, Iksan, Jeonbuk 570-749, Republic of Korea E-mail: ssimi@wku.ac.kr

${ }^{*}$ Contributed equally

Key words: $\beta$-lactam, aminoglycoside antibiotics, iuyeolin, methicillin-resistant Staphylococcus aureus

\section{Introduction}

Staphylococcus aureus (S. aureus), a bacterium that grows in the human nose and skin and a major pathogen for skin and soft-tissue infections, has previously been treated with methicillin. S. aureus is a gram-positive bacterium and a major nosocomial agent, responsible for several hospital-acquired infections, including bacteremia, skin infections and septic shock (1-3). Hospital-acquired infections comprise a serious problem, particularly due to the increased prevalence of methicillin-resistant $S$. aureus (MRSA) (4,5). This pathogen is associated with various infectious diseases and has a mortality rate of $36-50 \%(6,7)$. With increasing resistance to various antibiotics, combination therapy appears to be a useful option, particularly in developing countries where antibiotic availability is limited (8-10). Furthermore, MRSA strains have been found to be resistant not only to $\beta$-lactam antibiotics, but also to fluoroquinolones and other types of antibiotics (8).

Luteolin (LUT), a polyphenolic flavonoid compound, is present in numerous plant groups, including Magnoliophyta, Pinophyta, Pteridophyta and Bryophyta species. Dietary sources of LUT include carrots, peppers, celery, olive oil, peppermint, thyme, rosemary and oregano. LUT is considered to have diverse biological benefits, including cardioprotective, antioxidant, anti-inflammatory and anticancer activities (11-14). In addition, LUT has exhibited a strong antiproliferative activity against various human cancer cell lines, such as lung cancer cell lines, and is a widely used ingredient in nutritional supplements $(13,14)$. However, the antimicrobial activity of LUT against $S$. aureus has not yet been fully elucidated, but merits investigation.

The aim of the present study was to investigate the potential of using LUT in combination with antibiotics as an alternative therapeutic regime to overcome the drug-resistance of MRSA strains, including reference strains and clinical isolates. 


\section{Materials and methods}

Reagents. Ampicillin (AM), oxacillin (OX), gentamicin (GT) and luteolin $(\geq 98.0 \%)$ were purchased from Sigma-Aldrich (St. Louis, MO, USA). The structure of luteolin is shown in Fig. 1.

Bacterial strains and growth conditions. Five clinical MRSA isolates (DPS-1 to -5) were obtained from 5 different patients admitted to the Wonkwang University Hospital (Iksan, South Korea), and two reference strains were acquired from American Type Culture Collection (ATCC; Manassas, VA, USA): S. aureus ATCC 33591 (MRSA) and S. aureus ATCC 25923 (methicillin-sensitive S. aureus; MSSA). Prior to each experiment, bacteria were stored in $30 \%$ glycerol and frozen at $-70^{\circ} \mathrm{C}$. They were then cultured in Mueller-Hinton broth (MHB) and Mueller-Hinton agar (MHA; Difco; BD Biosciences, Sparks, MD, USA) by incubating at $37^{\circ} \mathrm{C}$ for $24 \mathrm{~h}$.

Minimum inhibitory concentration (MIC). MICs were determined using the broth microdilution method according to the guidelines of the Clinical and Laboratory Standards Institute (15). Briefly, a microorganism suspension was prepared by growing bacteria in broth for $24 \mathrm{~h}$, and adjusting to a $0.5 \mathrm{McF}$ arland standard turbidity $\left(\sim 1.5 \times 10^{8} \mathrm{CFU} / \mathrm{ml}\right)$. Final inoculums were adjusted to $1.5 \times 10^{6} \mathrm{CFU} / \mathrm{ml}$. Serially diluted antibiotics and/or LUT were then incubated with the inoculum at $37^{\circ} \mathrm{C}$ for $18 \mathrm{~h}$. The MIC was the lowest effective concentration of antimicrobial agent. At the end of the incubation period, the wells were visually examined for turbidity. Cloudiness indicated that bacterial growth had not been inhibited at the concentration of antimicrobial agent in the medium.

Checkerboard dilution test. Synergistic combinations were investigated using the preliminary checkerboard method (16). The MIC was defined as the lowest drug concentration, either alone or in combination, that inhibited visible bacterial growth. In vitro interactions were quantified by the fractional inhibitory concentration (FIC) index, which was calculated as $\mathrm{FIC}=(\mathrm{MIC}$ of drug $\mathrm{A}$ in combination $/ \mathrm{MIC}$ of drug $\mathrm{A}$ alone) + (MIC of drug B in combination/MIC of drug B alone). FIC indices (FICI) were interpreted as follows: $\leq 0.5$, synergy; $<0.5-\leq 0.75$, partial synergy; $>0.75-\leq 1.0$, additive effect; $>1.0-\leq 4.0$, indifference; and $>4.0$, antagonism. Finally, the varying rates of synergy between two given agents were determined (17). All experiments were conducted in triplicate.

Methyl thiazolyl tetrazolium assay (MTT) colorimetric assay. An MTT colorimetric assay for rapid detection of the presence of bacteria was performed as previously described (18-20). Briefly, a stock solution of $5 \mathrm{mg} / \mathrm{ml}$ MTT (Sigma-Aldrich) was prepared in phosphate-buffered saline and kept at $-70^{\circ} \mathrm{C}$. A final concentration of $1 \mathrm{mg} / \mathrm{ml}$ MTT was used in the assay. Following incubation of the bacteria for $24 \mathrm{~h}$ at $37^{\circ} \mathrm{C}, 20 \mu \mathrm{l}$ yellow MTT was added to a 96-well microtiter plate and incubation was continued for an additional $20 \mathrm{~min}$. Blue color indicated the presence of bacteria.
Table I. Interpreted FICI response for LUT + AM combinations against MRSA and MSSA strains.

\begin{tabular}{|c|c|c|c|c|c|}
\hline \multirow[b]{3}{*}{ Strain } & \multicolumn{4}{|c|}{$\operatorname{MIC}(\mu \mathrm{g} / \mathrm{ml})$} & \multirow[b]{3}{*}{ FICI } \\
\hline & \multicolumn{2}{|c|}{ LUT } & \multicolumn{2}{|c|}{$\mathrm{AM}$} & \\
\hline & Alone & $+\mathrm{AM}$ & Alone & +LUT & \\
\hline ATCC 33591 & 62.5 & 3.9 & 500 & 250 & 0.562 \\
\hline ATCC 25923 & 62.5 & 3.9 & 15.63 & 0.975 & 0.125 \\
\hline DPS-1 & 62.5 & 3.9 & 1,000 & 62.5 & 0.125 \\
\hline DPS-2 & 62.5 & 3.9 & 1,000 & 62.5 & 0.125 \\
\hline DPS-3 & 62.5 & 3.9 & 250 & 15.63 & 0.125 \\
\hline DPS-4 & 62.5 & 3.9 & 250 & 15.63 & 0.125 \\
\hline DPS-5 & 62.5 & 3.9 & 125 & 7.81 & 0.125 \\
\hline
\end{tabular}

FICI, fractional inhibitory concentration index; LUT, luteolin; AM, ampicillin; MRSA, methicillin-resistant Staphylococcus aureus; MSSA, methicillin-sensitive S. aureus; MIC, minimum inhibitory concentration.<smiles>O=c1cc(-c2ccc(O)c(O)c2)oc2cc(O)cc(O)c12</smiles>

Figure 1. Structure of luteolin.

Time-kill curve assay. A time-kill curve assay was performed as previously described (21) to study the effects of antimicrobial agent concentration on bacterial growth over time. A standard inoculum of $\sim 10^{6} \mathrm{CFU} / \mathrm{ml}$ was used. LUT (0.5 MIC) was used with antibiotics (0.5 MIC) in various combinations. A test plate containing only MHB plus inoculum served as the control. Viable strains were counted at different intervals up to $24 \mathrm{~h}$ at $37^{\circ} \mathrm{C}$. The rate and extent of bacterial death were determined by plotting viable colony counts $(\mathrm{CFU} / \mathrm{ml})$ against time in MHA. All experiments were conducted in triplicate.

Statistical analysis. All experiments were performed at least in triplicate. The data obtained from the experiments are presented as the mean \pm standard error. The statistical analysis was performed using one-way analysis of variance followed by Dunnett's t-test (SPSS software 19.0; IBM Corp., Armonk, NY, USA). $\mathrm{P}<0.01$ was considered to indicate a statistically significant difference.

\section{Results}

MICs and synergistic effect. Against all strains, the MIC of LUT was $62.5 \mu \mathrm{g} / \mathrm{ml}$. The synergistic effects of LUT with 
Table II. Interpreted FICI response for LUT + OX combinations against MRSA and MSSA strains.

\begin{tabular}{|c|c|c|c|c|c|}
\hline \multirow[b]{3}{*}{ Strain } & \multicolumn{4}{|c|}{$\operatorname{MIC}(\mu \mathrm{g} / \mathrm{ml})$} & \multirow[b]{3}{*}{ FICI } \\
\hline & \multicolumn{2}{|c|}{ LUT } & \multicolumn{2}{|c|}{ OX } & \\
\hline & Alone & $+\mathrm{OX}$ & Alone & +LUT & \\
\hline ATCC33591 & 62.5 & 3.9 & 500 & 31.25 & 0.125 \\
\hline АТCC25923 & 62.5 & 3.9 & 62.5 & 3.9 & 0.125 \\
\hline DPS-1 & 62.5 & 3.9 & 125 & 15.63 & 0.187 \\
\hline DPS-2 & 62.5 & 3.9 & 500 & 31.25 & 0.125 \\
\hline DPS-3 & 62.5 & 62.5 & 1,000 & 1,000 & 2 \\
\hline DPS-4 & 62.5 & 3.9 & 1,000 & 250 & 0.312 \\
\hline DPS-5 & 62.5 & 3.9 & 500 & 31.25 & 0.125 \\
\hline
\end{tabular}

FICI, fractional inhibitory concentration index; LUT, luteolin; OX, oxacillin; MRSA, methicillin-resistant Staphylocuccus aureus; MSSA, meticillin-sensitive S. aureus; MIC, minimum inhibitory concentration.

Table III. Interpreted FICI response for LUT + GT combinations against MRSA and MSSA strains.

\begin{tabular}{|c|c|c|c|c|c|}
\hline \multirow[b]{3}{*}{ Strain } & \multicolumn{4}{|c|}{$\operatorname{MIC}(\mu \mathrm{g} / \mathrm{ml})$} & \multirow[b]{3}{*}{ FICI } \\
\hline & \multicolumn{2}{|c|}{ LUT } & \multicolumn{2}{|c|}{ GT } & \\
\hline & Alone & +GT & Alone & + LUT & \\
\hline ATCC33591 & 62.5 & 3.9 & 62.5 & 3.9 & 0.125 \\
\hline ATCC25923 & 62.5 & 3.9 & 62.5 & 3.9 & 0.125 \\
\hline DPS-1 & 62.5 & 3.9 & 250 & 62.5 & 0.312 \\
\hline DPS-2 & 62.5 & 31.25 & 500 & 31.25 & 0.562 \\
\hline DPS-3 & 62.5 & 3.9 & 500 & 31.25 & 0.125 \\
\hline DPS-4 & 62.5 & 3.9 & 500 & 31.25 & 0.125 \\
\hline DPS-5 & 62.5 & 3.9 & 500 & 62.5 & 0.187 \\
\hline
\end{tabular}

FICI, fractional inhibitory concentration index; LUT, luteolin; GT, gentamicin; MRSA, methicillin-resistant Staphylococcus aureus; MSSA, meticillin-sensitive S. aureus; MIC, minimum inhibitory concentration.

various antibiotics were evaluated in MRSA strains using a checkerboard dilution assay. The antibacterial effects of LUT alone, each antibiotic alone, and LUT combined with one of the three antibiotics (AM, OX and GT) are shown in Tables I-III. The presence of LUT reduced the MIC of these antibiotics markedly against $S$. aureus strains. All strains were found to be resistant to AM, OX and GT, with MIC values ranging from 15.63 to $1,000 \mu \mathrm{g} / \mathrm{ml}$. LUT + antibiotic combinations exhibited markedly decreased MICs. In combination with LUT, the MICs of AM, OX and GT underwent 2-16-, 0-16- and 4-16-fold reductions, respectively. None of the aforementioned combinations exhibited an antagonistic effect. These findings demonstrate the promising potential of

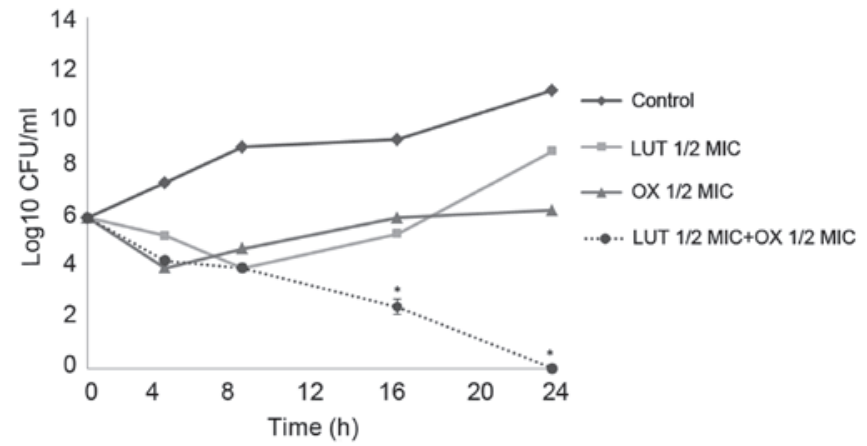

Figure 2. Time-kill curves of LUT and OX against a clinical MRSA strain (DPS-2). Data represent the mean of three independent experiments. "P $<0.001$ vs. OX alone. LUT, luteolin; OX, oxacillin; MRSA, methicillin-resistant Staphylococcus aureus; MIC, minimum inhibitory concentration.
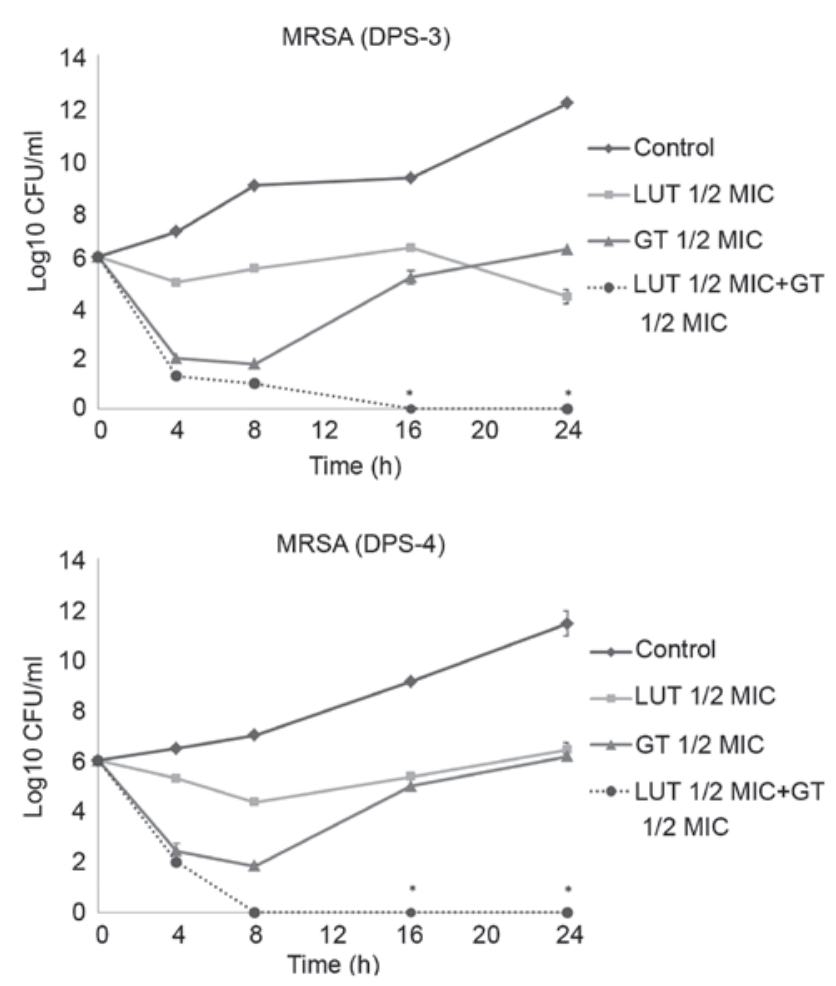

Figure 3. Time-kill curves of LUT and GT against clinical MRSA strains (DPS-3 and DPS-4). Data represent the mean of three independent experiments. "P<0.001 vs. GT alone. LUT, luteolin; GT, gentamicin; MRSA, methicillin-resistant Staphylococcus aureus. MIC, minimum inhibitory concentration.

LUT plus antibiotic combination therapy in the suppression of MRSA growth.

The combined use of LUT and AM, OX or GT antibiotics against the ATCC 33591 MRSA strain resulted in a FICI of 0.125 or 0.562 (Tables I-III). None of the combinations exhibited an antagonistic effect (FICI, >4.0). These results demonstrate that combinations of LUT with antibiotics could be used for the suppression of MRSA growth.

Time-kill curve assay. Time-kill tests were performed to examine the synergistic effects of LUT and antibiotics over time. The control did not exhibit a reduction in CFU counts, and LUT or antibiotic alone did not induce cell death after $24 \mathrm{~h}$. 
When used together, LUT and antibiotics markedly reduced bacterial counts. As shown in Figs. 2 and 3, the combination of $1 / 2$ MIC LUT $+1 / 2$ MIC antibiotic completely Notably, the combination of $1 / 2$ MIC LUT $+1 / 2$ MIC GT, completely inhibited the growth of $S$. aureus after $16 \mathrm{~h}$.

\section{Discussion}

The resistance of $S$. aureus to drugs, including synthetic penicillin and other conventional antibiotics, is a major obstacle in the treatment MRSA infection, which is an increasingly common type of infection, particularly among hospitalized patients (22). While vancomycin is often used as the only remaining effective antibiotic against MRSA, even vancomycin-resistant species of $S$. aureus have now been reported $(22,23)$. In the absence of any effective antibiotic treatment for multi-resistant infections, the development of alternative methods for the prevention and treatment of these diseases is required (22-24).

A consequence of the increase in the prevalence of resistant bacteria is that novel antimicrobial drugs active against infectious diseases are urgently required. Novel antimicrobial agents with no associated toxic or side effects may be developed from natural products. Combination therapy is the most commonly recommended empirical treatment for bacterial infections in intensive care units, since not all potential pathogens are susceptible to monotherapy; in addition, it may aid the prevention of antibacterial resistance (25). The aim of the present study was to investigate combination therapy with LUT and antibiotics against S. aureus, in order to find a solution to the problem of multi-drug resistance.

To the best of our knowledge, this is the first study investigating the potential of using antibiotics in combination with LUT in the treatment of MRSA and MSSA. As an indicator of the potency of LUT and antibiotic combinations against resistant strains of $S$. aureus, MIC values were determined. The antimicrobial activity of LUT was found to be moderate (MIC, $62.5 \mu \mathrm{g} / \mathrm{ml}$ ). Checkerboard dilution tests were performed to determine the action of LUT alone, as well as its synergistic action with antibiotics against the 7 strains. Although LUT alone had only a moderate inhibitory effect on MRSA growth, when a non-growth inhibitory dose of LUT $(62.5 \mu \mathrm{g} / \mathrm{ml})$ or antibiotic was used, the combination was shown to be highly effective, with a FICI of 0.125-0.562. Similar effects were observed in the MSSA strain. This experimental method clearly showed that LUT was a potent MRSA growth inhibitor. The use of three different antibiotics, namely AM, OX and GT, in combination with LUT clearly suppressed MRSA growth, enabling the dose of the antibiotics to be reduced. The time-kill curves confirmed the ability of LUT to increase the antibacterial effects of the antibiotics, synergistically reducing the bacterial counts below the lowest detectable limit after 8-24 h. The present study demonstrated the potential of LUT as an effective therapeutic agent against MRSA, reinforcing the possibility of substantially reducing the use of existing antibiotics. The results obtained for the combinations tested in the current study suggest that LUT can increase susceptibility to antibacterial action, as well as reduce the inducible antibiotic resistance of bacteria.
In conclusion, LUT may have potential as an antibacterial drug candidate for clinical use against MRSA and MSSA. The results of the present study are promising and may support the use of drugs derived from natural products.

\section{Acknowledgements}

This study was supported by the Basic Science Research Program through the National Research Foundation of Korea, the Ministry of Education, Science and Technology (grant nos. 2012-0004337 and 2008-0062484), and the Cooperative Research Program for Agriculture Science \& Technology Development (project no. PJ00962202), Rural Development Administration, Republic of Korea.

\section{References}

1. National Nosocomial Infections Surveillance System: National Nosocomial Infections Surveillance (NNIS) System Report, data summary from January 1992 to June 2002, issued August 2002. Am J Infect Control 30: 458-475, 2002.

2. Selvey LA, Whitby M and Johnson B: Nosocomial methicillin-resistant Staphylococcus aureus bacteremia: Is it any worse than nosocomial methicillin-sensitive Staphylococcus aureus bacteremia? Infect Control Hosp Epidemiol 21: 645-648, 2000.

3. López-Cortés LE, Gálvez-Acebal J, Del Toro MD, Velasco C, de Cueto M, Caballero FJ, Muniain MA, Pascual A and Rodríguez-Baño J: Effect of statin therapy in the outcome of bloodstream infections due to Staphylococcus aureus: A prospective cohort study. PLoS One 8: e82958, 2013.

4. Lowy FD: Staphylococcus aureus infections. N Engl J Med 339: 520-532, 1998.

5. DeLeo FR, Otto M, Kreiswirth BN and Chambers HF: Community-associated meticillin-resistant Staphylococcus aureus. Lancet 375: 1557-1568, 2010.

6. Baltch AL, Ritz WJ, Bopp LH, Michelsen PB and Smith RP: Antimicrobial activities of daptomycin, vancomycin, and oxacillin in human monocytes and of daptomycin in combination with gentamicin and/or rifampin in human monocytes and in broth against Staphylococcus aureus. Antimicrob Agents Chemother 51: 1559-1562, 2007.

7. Dancer SJ: The effect of antibiotics on methicillin-resistant Staphylococcus aureus. J Antimicrob Chemother 61: 246-253,2008.

8. Aqil F, Ahmad I and Owais M: Evaluation of anti-methicillin-resistant Staphylococcus aureus (MRSA) activity and synergy of some bioactive plant extracts. Biotechnol $\mathrm{J} \mathrm{1}$ : 1093-1102, 2006.

9. Miranda-Novales G, Leaños-Miranda BE, Vilchis-Pérez M and Solórzano-Santos F: In vitro activity effects of combinations of cephalothin, dicloxacillin, imipenem, vancomycin and amikacin against methicillin-resistant Staphylococcus spp. strains. Ann Clin Microbiol Antimicrob 5: 25, 2006.

10. Kastoris AC, Rafailidis PI, Vouloumanou EK, Gkegkes ID and Falagas ME: Synergy of fosfomycin with other antibiotics for Gram-positive and Gram-negative bacteria. Eur J Clin Pharmacol 66: 359-368, 2010.

11. López-Lázaro M: Distribution and biological activities of the flavonoid luteolin. Mini Rev Med Chem 9: 31-59, 2009.

12. Lin Y, Shi R, Wang X and Shen HM: Luteolin, a flavonoid with potential for cancer prevention and therapy. Curr Cancer Drug Targets 8: 634-646, 2008.

13. Seelinger G, Merfort I and Schempp CM: Anti-oxidant, anti-inflammatory and anti-allergic activities of luteolin. Planta Med 74: 1667-1677, 2008.

14. Seelinger G, Merfort I, Wölfle U and Schempp CM: Anti-carcinogenic effects of the flavonoid luteolin. Molecules 13: 2628-2651, 2008.

15. Clinical and Laboratory Standards Institute (CLSI): Methods for dilution antimicrobial susceptibility tests for bacteria that grow aerobically; approved standard. CLSI Document M7-A7. 7th edition. CLSI, Wayne, PA, USA, 2006.

16. Odds FC: Synergy, antagonism, and what the chequerboard puts between them. J Antimicrob Chemother 52: 1, 2003. 
17. Mazumdar K, Dutta NK, Kumar KA and Dastidar SG: In vitro and in vivo synergism between tetracycline and the cardiovascular agent oxyfedrine $\mathrm{HCl}$ against common bacterial strains. Biol Pharm Bull 28: 713-717, 2005.

18. Scheuber PH, Mossmann H, Beck G and Hammer DK: Direct skin test in highly sensitized guinea pigs for rapid and sensitive determination of staphylococcal enterotoxin B. Appl Environ Microbiol 46: 1351-1356, 1983.

19. Abate G, Mshana RN and Miörner H: Evaluation of a colorimetric assay based on 3-(4,5-dimethylthiazol-2-yl)-2,5-diphenyl tetrazolium bromide (MTT) for rapid detection of rifampicin resistance in Mycobacterium tuberculosis. Int J Tuberc Lung Dis 2: 1011-1016, 1998.

20. Shi YJ, Chen J and Xu M: A new method for antimicrobial susceptibility testing of in vitro-cultured bacteria by means of resonance light scattering technique. J Microbiol Biotechnol 18: 118-123, 2008.

21. Chang SC, Chen YC, Luh KT and Hsieh WC: In vitro activities of antimicrobial agents, alone and in combination, against Acinetobacter baumannii isolated from blood. Diagn Microbiol Infect Dis 23: 105-110, 1995.
22. Roth DM, Senna JP and Machado DC: Evaluation of the humoral immune response in BALB/c mice immunized with a naked DNA vaccine anti-methicillin-resistant Staphylococcus aureus. Genet Mol Res 5: 503-512, 2006.

23. Pearson H: 'Superbug' hurdles key drug barrier. Nature 418: 469 , 2002.

24. Ohwada A, Sekiya M, Hanaki H, Arai KK, Nagaoka I, Hori S, Tominaga S, Hiramatsu K and Fukuchi Y: DNA vaccination by mecA sequence evokes an antibacterial immune response against methicillin-resistant Staphylococcus aureus. J Antimicrob Chemother 44: 767-774, 1999.

25. Drago L, De Vecchi E, Nicola L and Gismondo MR: In vitro evaluation of antibiotics' combinations for empirical therapy of suspected methicillin resistant Staphylococcus aureus severe respiratory infections. BMC Infect Dis 7: 111, 2007. 